Revue d'histoire de l'Amérique française

RAS REVUE D.HISTOIRE DE L'AMÉRIQUE FRANÇAISE

\title{
Le milieu d'origine du clergé québécois 1775-1840 : mythes et réalités
}

\section{Serge Gagnon et Louise Lebel-Gagnon}

Volume 37, numéro 3, décembre 1983

URI : https://id.erudit.org/iderudit/304178ar

DOI : https://doi.org/10.7202/304178ar

Aller au sommaire du numéro

Éditeur(s)

Institut d'histoire de l'Amérique française

ISSN

0035-2357 (imprimé)

1492-1383 (numérique)

Découvrir la revue

Citer cet article

Gagnon, S. \& Lebel-Gagnon, L. (1983). Le milieu d'origine du clergé québécois 1775-1840 : mythes et réalités. Revue d'histoire de l'Amérique française, 37(3), 373-397. https://doi.org/10.7202/304178ar d'utilisation que vous pouvez consulter en ligne.

https://apropos.erudit.org/fr/usagers/politique-dutilisation/ 


\title{
LE MILIEU D'ORIGINE DU CLERGÉ QUÉBÉCOIS 1775-1840: MYTHES ET RÉALITÉS
}

\author{
SERGE GAGNON* \\ LOUISE LEBEL-GAGNON \\ Département des sciences humaines \\ Université du Québec à Trois-Rivières
}

\begin{abstract}
Le mythe tenace d'un clergé d'Ancien Régime sortant des couches populaires a maintenant largement éclaté. T. Tackett, «L'histoire sociale du clergé diocésain dans la France du XVIII ${ }^{\mathrm{e}}$ siècle», RHMC (avril-juin 1979): 209.
\end{abstract}

À une époque où, au Québec, quatre habitants sur cinq vivaient hors des villes, d'où venaient les prêtres? De la ville ou de la campagne? Y a-t-il eu, au cours des ans, mutation de la géographie du recrutement? Depuis la magistrale étude du géographe Hamelin, on se réfère implicitement à ses conclusions présumées valables pour les siècles antérieurs au nôtre: «Sur le plan de l'origine spatiale, le prêtre a beaucoup plus été, dans le passé, l'homme de plaine rurale du Saint-Laurent... que celui des villes.»' ${ }^{1}$ 'intuition est-elle conforme à la réalité pour l'ensemble des XVIII ${ }^{\mathrm{e}}$ et $\mathrm{XIX}^{\mathrm{e}}$ siècles? Après avoir répondu à cette première interrogation, nous nous attaquerons à une question plus délicate: l'origine sociale, appréhendée par la mention de l'occupation paternelle. La réponse à cette question est d'autant plus difficile que nous ne possédons pas au Québec de codification socio-professionnelle opératoire pour les travaux sur le passé lointain. Néanmoins, quelque modeste qu'elle soit, toute réponse provisoire, voire incomplète, à ce problème précisera ce que nous croyons savoir sur le sujet. Jean-Pierre Wallot a écrit à propos du début du $\mathrm{XIX}^{\mathrm{e}}$ siècle: «l'Église puise ses effectifs surtout dans les milieux paysan, artisanal ou professionnel.» ${ }^{2}$ L'observation a peu de chance d'être fausse; mais on sait qu'il importe de savoir dans quelle proportion, paysannerie, boutiquiers, artisans et professionnels ont donné des fils à l'Église.

* Nous remercions le Conseil des Arts du Canada qui a financé le début de cette recherche commencée il y a plus de 10 ans; son aide nous a permis d'obtenir le service d'un assistant de recherche comme il s'en trouve rarement, Claude Bélanger. Nous l'en remercions. Les universités d'Ottawa et du Québec ont dégagé le chercheur principal pour poursuivre la quête de renseignements et la rédaction. Merci à Lucie Lainesse et Pauline Tremblay qui, patiemment, avec sagacité, ont su déchiffrer nos grimoires. Toujours empressés à répondre à nos requêtes, les archivistes des archevêchés de Québec et de Montréal ont droit à une mention spéciale de reconnaissance. Merci à Jean Roy qui nous a familiarisés avec les travaux européens.

1 «Évolution numérique séculaire du clergé catholique dans le Québec», Recherches sociographiques, 2 (avril-juin 1961): 213-34.

2 J.-P. Wallot, Un Québec qui bougeait (Montréal, Boréal Express, 1973), 217.

RHAF, vol. 37, no 3, décembre 1983 
Les limites chronologiques retenues pour l'enquête exigent quelque explication. On pourrait penser que la date de 1775 a été choisie au seuil d'un nouveau régime constitutionnel ou encore pour éliminer l'époque de la Conquête, période peu propice à une lecture de longue durée. En réalité, des considérations beaucoup plus prosaïques ont fixé la date de démarrage. Il existait aux archives de l'archevêché de Québec une série de titres cléricaux débutant en 1775 . Or ces actes notariés nous le verrons - sont la principale source de renseignements sur l'origine sociale des prêtres.

Dans l'ensemble, la période est marquée par une pénurie de pasteurs. Pour expliquer la disette, les auteurs ont invoqué la tiédeur religieuse des humbles et le détachement de la petite bourgeoisie. Quelles qu'en soient les causes, la pénurie se traduit par des difficultés multiples à assurer un minimum d'encadrement dans les paroisses. La croissance naturelle de la population franco-catholique est très rapide; celle-ci double tous les 25 ou 30 ans cependant que le nombre de prêtres reste stable pendant une longue période. Le nombre de catholiques par prêtre passe de 750 en 1780 à 1834 en $1830^{3}$. Sans la venue d'une cinquantaine de recrues françaises, réfugiés de la Révolution, la pénurie aurait atteint des proportions encore plus grandes. Les prêtres d'origine française représentent près du tiers de tout le clergé en service au Québec, au début du XIX ${ }^{\mathrm{e}}$ siècle. Tout change à partir des années 1830-40. D'abord modeste, la croissance «naturelle» du nombre de vocations va rapidement atteindre des proportions record. Deux ou trois décennies plus tôt, les communautés de prêtres réguliers ont à toutes fins utiles disparu du Québec. Après la Conquête, le gouvernement britannique à décrété la suppression des communautés d'hommes; le dernier jésuite meurt en 1800 , le dernier récollet, en 1813. En conséquence, le clergé québécois est presque uniquement composé de séculiers au cours des premières décennies du siècle dernier. La pénurie, l'absence de réguliers, la «canadianisation» sont autant d'éléments qui donnent un relief particulier de la période à l'étude.

Il est une dernière caractéristique de cette analyse, dont il faut saisir le lecteur dès l'introduction. À plusieurs reprises, nous recourons aux procédés de l'étude comparative. Il nous est apparu, à la lecture de l'historiographie française, que le cheminement québécois en matière de recrutement sacerdotal n'avait rien de singulier. Nous espérons que ces comparaisons serviront de points de repère au vaste chantier de l'histoire comparée de la société rurale de la France de l'Ouest et du Québec mis sur pied il y a quelques années et dont nous récoltons depuis peu les premiers fruits ${ }^{4}$.

\footnotetext{
3 Hamelin, art. cit., 225.

4 En coll. Société rurale dans la France de l'Ouest et au Québec (XVIII $-X X^{e}$ siècles) (Université de Montréal, EHESS, 1981).
} 


\section{I- La construction d'une statistique}

\section{a) Le mouvement des ordinations}

La série chronologique d'ordinations construite pour la présente recherche a été constituée à l'aide du Dictionnaire biographique de Allaire, puis confrontée aux renseignements du Répertoire Tanguay ${ }^{5}$. Pour les années 1775-1825, la liste nominative ainsi obtenue a été validée par la consultation des actes de prêtrise inscrits au Registre des insinuations ecclésiastiques. Ces tests nous assurent que la statistique du nombre annuel d'ordinations n'est pas très loin de la réalité. La marge d'erreur est probablement inférieure à $5 \%$.

La liste des ordinands servant de base à l'enquête ne comprend pas tous les prêtres qui ont reçu le sacrement de l'ordre de l'Évêque de Québec. Quelques recrues originaires des Maritimes et de l'Ontario sont exclues. Il en est de même pour des prêtres d'origine américaine, britannique ou française. Ceux-ci ont été écartés puisqu'il s'agit de mesurer le nombre et la provenance des prêtres issus de la majorité catholique et française. Par contre, les clercs d'origine québécoise qui ont fait une partie de leur carrière hors Québec sont inclus. En somme, le groupe retenu correspond à peu près à ce que Louis-Edmond Hamelin a appelé le clergé franco-québécois au sens strict: «un clergé exclusivement québécois de langue française... groupant les prêtres nés dans le Québec de parents canadiens-français et oeuvrant dans le Québec auprès de catholiques de langue française. ${ }^{6}$ Une dernière précision s'impose. Contrairement à la coutume, les séries chronologiques sont construites par tranches de six ans. Cette singularité a pour but de découper la période en classes chronologiques d'égale valeur afin de faciliter la comparaison dans le temps.

En prenant pour point de comparaison l'indice de croissance des années 1775-80, on ne peut s'empêcher d'observer la chute prononcée du début du XIX ${ }^{\mathrm{e}}$ siècle. Elle constitue, comme l'a noté Louis-Edmond Hamelin ${ }^{7}$, le creux du mouvement de recrutement. Dans l'état actuel des connaissances, il est difficile d'expliquer cette chute autrement que par des généralités sur l'affadissement de la fibre religieuse. On sait qu'elle est suffisante pour alarmer l'épiscopat ${ }^{8}$. En regard d'une population de fidèles qui s'accroît peut-être de $60 \%$ entre 1790 et 1810 , le nombre de prêtres affectés à la pastorale a tendance à se stabiliser autour

\footnotetext{
5 J.-B.-A. Allaire. Dictionnaire biographique du clergé canadien-français (Montréal et Saint-Hyacinthe, 1910-34, 6 vol.). - Cyprien Tanguay. Répertoire du clergé canadien (Montréal, 1893).

6 Hamelin, art. cit., 192.

«La production sacerdotale comparativement aux naissances n'a jamais été aussi basse», Hamelin, art. cit., 207.

Jean-Pierre Wallot rapporte de nombreux témoignages dans Un Québec qui bougeait (Boréal Express, 1973), 190ss.
} 
TABLEAU I

NOUVEAUX PRÊTRES D'ORIGINE FRANCO-QUÉBÉCOISE, 1775-18409

\begin{tabular}{l|c|c|c}
\hline & $\begin{array}{c}\text { Nombre } \\
\text { absolu }\end{array}$ & $\begin{array}{c}\text { Nombre de nouveaux } \\
\text { prêtres/année }\end{array}$ & \multicolumn{2}{|c}{$\begin{array}{c}\text { Indice de croissance } \\
(1775-80 \quad 100)\end{array}$} \\
\hline $1775-80$ & 33 & 5,5 & 100 \\
$1781-86$ & 35 & 5,83 & 106,6 \\
$1787-92$ & 38 & 6,33 & 115,15 \\
$1793-98$ & 30 & 5,00 & 90,9 \\
$1799-04$ & 19 & 3,16 & 57,5 \\
$1805-10$ & 25 & 4,16 & 75,75 \\
$1811-16$ & 39 & 6,5 & 118,18 \\
$1817-22$ & 46 & 7,66 & 139,39 \\
$1823-28$ & 66 & 11,0 & 200,0 \\
$1829-34$ & 70 & 11,66 & 212,12 \\
$1835-40$ & 83 & 13,83 & 251,51 \\
\hline
\end{tabular}

Source: Dictionnaire biographique du clergé, de Allaire.

de 150 . Ce sont les prêtres enseignants qui augmentent en nombre, passant de la douzaine vers 1800 à plus du double dix ans plus $\operatorname{tard}^{10}$.

Sans la venue de prêtres français, le Québec se serait trouvé vers 1800 dans une situation comparable à celle de la France révolutionnaire, nonobstant l'absence au Québec d'un quelconque mouvement de défection. En dépit de l'apport étranger, la situation apparaît en effet aussi précaire que dans l'ancienne métropole, à la veille de la Restauration. Là-bas, le clergé est alors très âgé. En 1809, un prêtre sur trois a soixante ans et plus. Ici, la situation n'est pas aussi critique, mais elle suit une même trajectoire ${ }^{11}$. La correspondance épiscopale du début du $\mathrm{XIX}^{\mathrm{e}}$ siècle regorge de plaintes. L'évêque ne cesse d'espérer un redressement qui tarde à venir. Comme en France, mais pour des motifs différents, on ne parvient pas à satisfaire la demande pendant plusieurs années. Outre-mer, il n'y a à peu près pas eu d'ordinations durant la

\footnotetext{
9 Nos statistiques diffèrent quelque peu de celles établies par James Lambert dans Monseigneur, the Catholic Bishop Joseph Octave Plessis, Church, State and Society in Lower Canada (PhD, Université Laval, 1981), 432-33 et 517-20. Lambert a retenu, comme Allaire son guide, les prêtres venus de Grande-Bretagne ou des États-Unis, qui ont fait du ministère auprès des Canadiens. Il a aussi vraisemblablement rattaché au groupe quelques unités provenant des milieux francophones hors Québec. Il écrit en note, p. 520: «The difference between Hamelin's figures («Nombre annuel de nouveaux prêtres, Canada français (1600-1933)», Bulletin des recherches historiques, avril-juin 1959: 35-44) and my own is due to the fact that Hamelin's are based on Cyprien Tanguay... while mine are based on Allaire... (who) counted... Canadian and English Priests who ministered to Canadians».

10 Sur la répartition annuelle des effectifs entre les fonctions éducative et pastorale, voir le tableau de Wallot, op . cit., 192-93.

11 Voici, d'après les statistiques de James Lambert (op. cit., 665), l'évolution du pourcentage de prêtres de 51 ans et plus: 1790: 23\%; 1800: 18,1\% (l'abaissement est dû peut-être à la venue de prêtres étrangers); $1815: 37,9 \% ; 1820: 36,5 \% ; 1825: 36,1 \%$.
} 
Révolution. À la veille de la Restauration, on estime au quart les communautés de fidèles sans encadrement clérical. Ici, Plessis est aux prises avec un problème de pénurie de même ampleur. C'est que contrairement aux diocèses français, la population catholique s'accroît à un rythme exponentiel; au cours des années 1830 , le rapport prêtre/fidèles dépasse, nous l'avons déjà noté, le chiffre de 1800 par pasteur, même si le nombre de prêtres s'accroît. L'encadrement religieux n'a jamais été aussi faible au Québec ni avant, ni après cette période. En France, le nombre d'ordinations dépasse celui des décès de prêtres à partir de 1825; on observe un renversement de tendance analogue au Québec vers le même moment. Ici, l'évêque peut compter sur une croissance sensible du nombre de vocations à partir des années 1825-30. Au moment de la Rébellion, le nombre de prêtres en exercice oscille autour de 300, le double du nombre de pasteurs actifs un quart de siècle plus tôt, même si le contingent de réfugiés français n'est plus. La moyenne annuelle de recrues québécoises dépasse l'indice de base des années 1775-80 à partir de 1811-16. L'indice qui est alors de 118 passe à 200 au cours de la période 1823-28. Il atteint 250 au cours de la dernière classe chronologique (1835-40). La croissance «naturelle» des vocations combinée à l'apport de nouveaux contingents français après 1840 va ramener le rapport prêtre/fidèles à 1 pour 1000 au milieu du XIX ${ }^{e}$ siècle.

\section{b) L'origine spatiale}

L'esquisse du mouvement des vocations québécoises fournit un point de repère pour construire une géographie du recrutement ecclésiastique: le diocèse importe-t-il des recrues, en envoie-t-il à l'extérieur? Le découpage du diocèse en sous-espaces offre-t-il des contrastes quant au nombre absolu et relatif de vocations? La ville est-elle plus «féconde» que la campagne ou vice versa? Voyons d'abord comment les vocations sont distribuées dans l'espace québécois.

Le territoire est alors subdivisé en trois régions administratives appelées districts. Les districts de Québec, de Trois-Rivières, de Montréal $^{12}$ constituent une bonne base pour ventiler l'origine spatiale. L'évêque lui-même renvoie à ces régions administratives dans sa correspondance de gestion. L'origine géographique des ordinands nous est

12 À sa frontière est, le district de Montréal s'arrête à Saint-Barthélémy, aujourd'hui dans le diocèse de Joliette, et à Sorel sur la rive sud du Saint-Laurent. Le district de Trois-Rivières est délimité à l'ouest par les paroisses de Maskinongé et de Saint-Michel d'Yamaska, à l'est par Sainte-Anne-de-la-Pérade et Saint-Pierre-les-Becquets. Il occupe à peu près la surface aujourd'hui administrée par les sièges épiscopaux de Trois-Rivières et de Nicolet. Le district de Québec commence aux Grondines et à Deschaillons, respectivement situées sur les rives nord et sud du Saint-Laurent. La frontière est du district est difficile à préciser à cause de la formation des districts de Gaspé et plus tard de Rimouski. Le district de Gaspé est d'autant plus facilement exclu des calculs qu'il ne donne aucun prêtre à l'Église; il compte 13312 habitants en 1831, partagé également entre catholiques et protestants. La recrue la plus à l'est vient de Trois-Pistoles, à la toute fin de la période. 
connue pour $90 \%$ de la population étudiée (438/484). C'est plus de garantie qu'il n'en faut pour accréditer la valeur de la statistique.

La répartition du nombre de vocations par districts présente des écarts significatifs. Ceux-ci prennent encore plus de relief lorsqu'on tient compte des populations respectives de chacune des trois régions administratives. Pour estimer la «fécondité différentielle» des régions, nous comptons deux estimés de population assez fiables: ceux des recensements de 1790 et de 1831 .

L'écart de population entre le district de Québec et celui de Montréal s'accroît sensiblement avec le temps. C'est dire que pour l'ensemble de la période, le district de Québec, avec 241 vocations, est environ

TABLEAU 2

RÉPARTITION DU NOMBRE DE VOCATIONS PAR DISTRICTS

(QUÉBEC 1775-1840)

\begin{tabular}{l|c|c|c}
\hline Périodes & District de Québec & District de Montréal & $\begin{array}{c}\text { District de } \\
\text { Trois-Rivières }\end{array}$ \\
\hline $1775-1780$ & 17 & 8 & 0 \\
$1781-86$ & 15 & 18 & 0 \\
$1787-92$ & 23 & 12 & 1 \\
$1793-98$ & 14 & 12 & 1 \\
$1799-04$ & 10 & 8 & 0 \\
$1805-10$ & 10 & 5 & 2 \\
$1811-16$ & 15 & 12 & 6 \\
$1817-22$ & 20 & 22 & 3 \\
$1823-28$ & 36 & 17 & 8 \\
$1829-34$ & 33 & 19 & 10 \\
$1835-40$ & 48 & 158 & 39 \\
\hline Total & 241 & 19 & 8 \\
\hline
\end{tabular}

TABLEAU 3

ESTIMÉS DE LA POPULATION DU QUÉBEC PAR DISTRICTS EN 1790 ET $1830^{13}$

\begin{tabular}{l|c|c|c}
\hline Année & District de Québec & District de Montréal & $\begin{array}{c}\text { District de } \\
\text { Trois-Rivières }\end{array}$ \\
\hline 1790 & 61000 & 84000 & 16000 \\
1830 & 152000 & 280000 & 57000 \\
\hline
\end{tabular}

13 Les sources imprimées à partir desquelles sont estimées les populations, chiffres arrondis, sont: 1) Canada, Census of Canada 1665-1871, (Ottawa, 1876), IV: 95; 2) Almanach de Québec, année 1827, appendice; voir aussi Louis Rousseau, La prédication à Montréal 18001830 (Montréal, Fides, 1978), 74; Ivanhoë Caron, La colonisation de la province de Québec (Québec, L'Action sociale, 1923), 271-74. 
deux fois plus «fertile» que la région administrative montréalaise; celleci ne forme que 158 nouveaux prêtres, en dépit de sa population supérieure. Le contraste, au surplus, s'accentue avec le temps. Quand le district de Montréal fournit un prêtre à l'Église pour les années 178198, celui de Québec en donne 1,23; entre 1822 et 1834, le rapport est de 1 à 1,39 , alors que la population du district montréalais est près du double de celle de la zone administrative de Québec.

À la fin du XVIII ${ }^{\mathrm{e}}$ siècle, le district central, celui de Trois-Rivières, est une région à faible densité de population. En revanche, celle-ci progresse plus rapidement que celle du district de Québec durant les premières décennies du XIX ${ }^{\mathrm{e}}$ siècle. Au total, cependant, le nombre de vocations issues de la région du centre du Québec est trop faible pour légitimer la comparaison avec les régions administratives est et ouest de la province. La répartition des vocations entre les recrues urbaines et rurales ne semble pas non plus pertinente pour le district de TroisRivières; la ville renferme 1200 habitants en 1790, un peu plus du double en 1830. Bien qu'elle réponde aux critères juridiques du cadre urbain, l'agglomération trifluvienne demeure une petite localité. Elle dispense certes des services hospitaliers, judiciaires et autres; mais par sa taille, elle se compare davantage aux villages qu'aux villes des districts voisins. Au reste, c'est seulement après la fondation du diocèse (1852) que la ville est dotée d'un collège-séminaire. Aussi peut-on constater sans étonnement que Trois-Rivières fournit moins de prêtres (3) que Nicolet (8), Baie-du-Febvre (6), Yamachiche (5) et SainteAnne-de-la-Pérade (4), toutes paroisses situées dans le district central à proximité de Trois-Rivières. Il faut peut-être voir dans cette distribution l'illustration d'une «surfécondité» rurale; mais laissons-là ce raisonnement appuyé sur de trop petits nombres et voyons plutôt comment se répartissent à cet égard populations et vocations des deux districts extrêmes de la province.

Sachant que la ville de Québec passe de 14000 à 28000 entre 1790 et 1830 , cependant que Montréal fait un bond de 18000 à 32000 au cours de la même période, il faut constater le tarissement progressif de Québec et de Montréal, comme bassins de recrutement sacerdotal. $\mathrm{Au} \mathrm{XIX}^{\mathrm{e}}$ siècle, Montréal ne donne pour ainsi dire plus de prêtres: 22 en 36 ans (1805-40), soit moins d'un par année. Québec, par contre, moins peuplé que Montréal, produit plus de prêtres que cette dernière; après la chute des années $1805-22$, on note une reprise au cours des années 1823-40: 2 prêtres par année, en moyenne. Il faut nuancer ces calculs effectués sur des populations globales, c'est-à-dire sans tenir compte de l'appartenance religieuse ${ }^{14}$. En 1830, la ville épiscopale

14 Rappelons que, au recensement de 1831, le district de Québec compte 151985 habitants, catholiques à $78,82 \%$; le district de Montréal, alors occupé par 290052 personnes compte 79,05\% de catholiques. 
TABLEAU 4

RÉPARTITION DES VOCATIONS ENTRE LA VILLE ET LA CAMPAGNE DANS LES DISTRICTS DE QUÉBEC ET DE MONTRÉAL, 1775-1840

\begin{tabular}{l|c|c|c|c|c|c}
\hline & \multicolumn{3}{|c|}{ District de Quépec } & \multicolumn{3}{c}{ District de Montréal } \\
Périodes & Ville & Campagne & Total & Ville & Campagne & Total \\
\hline $1775-80$ & 12 & 5 & 17 & 7 & 1 & 8 \\
$1781-86$ & 14 & 1 & 15 & 13 & 5 & 18 \\
$1787-92$ & 13 & 10 & 23 & 4 & 8 & 12 \\
$1793-98$ & 11 & 3 & 14 & 10 & 2 & 12 \\
$1799-04$ & 10 & 0 & 10 & 7 & 1 & 8 \\
$1805-10$ & 5 & 5 & 10 & 3 & 2 & 5 \\
$1811-16$ & 5 & 10 & 15 & 4 & 8 & 12 \\
$1817-22$ & 4 & 16 & 20 & 5 & 17 & 22 \\
$1823-28$ & 12 & 24 & 36 & 2 & 15 & 17 \\
$1829-34$ & 10 & 23 & 33 & 5 & 20 & 25 \\
$1835-40$ & 13 & 35 & 48 & 3 & 16 & 19 \\
\hline Total & 109 & 132 & 241 & 63 & 95 & 158 \\
\hline
\end{tabular}

compte 8000 non-catholiques, soit près de 30\% de l'ensemble. L'île de Montréal renferme une mosaïque religieuse présumément comparable à celle de la ville épiscopale ${ }^{15}$. Par ailleurs, comme la répartition de la population entre la ville et la campagne est demeurée à peu près constante tout au long de la période pour l'ensemble du Québec, il faut conclure à la mise en place progressive de la zone rurale comme lieu privilégié de recrutement.

Notons encore ici une notable différence entre le district de Montréal et celui de Québec. En 1830, la zone rurale de la région administrative de Québec compte 125000 habitants, celle du district de Montréal, le double. Le nombre de vocations rurales entre 1823 et 1834 est de 35 pour le district de Montréal, de 47, pour le district de Québec. Compte tenu de la taille respective des populations en cause, l'écart s'avère considérable. Il reflète sûrement la tiédeur religieuse de l'ouest du Québec, soulignée à maintes reprises dans la correspondance épiscopale. N'est-ce pas dans cette région qu'ont eu lieu les soulèvements de 1837-38, malgré l'opposition des prêtres? Durant les années 183540,16 jeunes de la campagne montréalaise reçoivent la prêtrise, en regard de 35 pour la zone rurale du district de Québec.

\footnotetext{
15 Au recensement de 1831 , le comté de Montréal, qui comprend la ville et les environs, renferme 43773 habitants répartis ainsi: 32500 catholiques, 10600 protestants. Robert Rumilly, Histoire de Montréal (Montréal, Fides, 1970), 2: 174.
} 
Sans que l'on puisse vraiment l'expliquer, tout se passe encore une fois un peu comme en France ${ }^{16}$, même si les changements structuraux en cause ne sont pas synchronisés. Une phase d'équilibre entre ville et campagne succède à une très nette surreprésentation rurale. Vers la fin du XVIII ${ }^{\mathrm{e}}$ siècle, les villes de Québec et de Montréal fournissent près de trois prêtres sur quatre à l'ensemble du Québec. En 1830-40, la proportion est inversée ${ }^{17}$. En somme, le curé d'Ars, fils d'une humble famille paysanne, ne semble pas être un prototype de recrutement exclusivement français. L'histoire comparée nous apprend qu'ici et peutêtre ailleurs, le prêtre catholique du XIX $\mathrm{X}^{\mathrm{e}}$ siècle est venu du plat pays, malgré quelques exceptions ${ }^{18}$ qui, par leur petit nombre, confirment la «loi» du recrutement rural.

16 Dans Essor ou déclin du clergé français? (Paris, Éditions du Cerf, 1950), le chanoine Fernand Boulard avait déjà pressenti le changement; l'Évêque de Quimper déclare en 1811: «Nous ne trouvons plus dans les classes supérieures de la société, des jeunes gens qui se destinent à l'état ecclésiastique. C'est dans la classe des pauvres cultivateurs que se réduisent toutes nos ressources» (cité op . cit., 117). Charles Pouthas écrit, dans L'Église et les questions religieuses: $1^{\text {er }}$ vol. 1814 1848 (CDU, Paris, s.d.), 168: «Il est visible que la masse du clergé français a une origine essentiellement populaire, il se recrute surtout dans la classe paysanne; il se recrute peu et de moins en moins dans les classes riches, bourgeoisie et noblesse. Ceci est plus sensible encore sous la Monarchie de Juillet que sous la Restauration. Sous la Restauration, l'entraînement des grandes familles de la noblesse vers l'Église, soit par tradition familiale, soit par conviction politique, alimente encore pendant cette période les séminaires; à partir de la monarchie de Juillet, au contraire, la noblesse, à son tour, se détache des fonctions ecclésiastiques.» Claude Langlois a observé «la désaffection des villes», dans Le diocèse de Vannes au XIX ${ }^{e}$ siècle, 1800-1830 (Paris, Klincksieck, 1974), 584. Un autre exemple breton confirme: Michel Lagrée, Mentalités, religion et histoire en Haute Bretagne au XIX $X^{e}$ siècle - le diocèse de Rennes 1815-1848 (Paris, Klincksieck, i977), 178-185; sur 224 prêtres ordonnés entre 1814 et $1848,82 \%$ sont d'origine rurale, $18 \%$ d'origine urbaine, ce qui coïncide avec la répartition rurale/urbaine de la population. Dans la France du nord-ouest de la fin du XIX ${ }^{\mathrm{e}}$ siècle, Hilaire a noté l'accentuation du déclin des zones urbaines, sensible à compter de la monarchie de Juillet; Yves-Marie Hilaire, Une chrétienté au $X I X^{e}$ siècle? La vie religieuse des populations du diocèse d'Arras (1840-1914), (Presses de l'Université de Lille III, 1977), 1: 292-95. Même prédominance rurale dans un diocèse du Midi, l'Hérault de Gérard Cholvy, «Les vocations sacerdotales et religieuses dans le diocèse de Montpellier (1801-1956)», Annales du Midi (1959), 2-3: 222-29.

Pour le XVIII ${ }^{\mathrm{e}}$ siècle, les références sont beaucoup plus nombreuses. Dans Les hommes, l'Église et Dieu dans la France du XVIII' siècle (Paris, Hachette, 1978), Jean Quéniart a synthétisé les résultats des diverses études régionales aux pages 297-306; dans la première moitié du XVIII siècle, la noblesse est surreprésentée par rapport à ses effectifs dans la population globale. Des classes moyennes de la ville, il écrit qu'elles ont une fécondité sacerdotale élevée: «Dans la plupart des diocèses, avocats, procureurs, gens de roi, ces milieux d'officiers ou de robins... constituent avec les marchands, groupe beaucoup plus flou, le terreau d'où sont issus la plupart des prêtres»; plus progressivement, désaffection des élites urbaines, noblesse et bourgeoisie, notamment à Aix, à Coutances, à Rennes; désertion des riches, tarissement des villes. Quéniart évoque une «ruralisation du recrutement... une plus grande participation des élites rurales au recrutement des prêtres»; au cours des décennies pré-révolutionnaires: «double mouvement de translation des vocations, des élites urbaines à la petite bourgeoisie, de la ville à la campagne... La place croissante des élites rurales dans les vocations sacerdotales annonce dès la fin de l'Ancien régime un type de recrutement qui caractérise le XIX ${ }^{\mathrm{e}}$ siècle». Timothée Tackett a repris, en le précisant, l'ensemble du dossier dans «L'histoire sociale du clergé diocésain dans la France du XVIII ${ }^{\mathrm{e}}$ siècle», Revue d'histoire moderne et contemporaine (avril-juin 1979): 198-234. de $21,7 \%$.

17 Pour 1775-1805, 71,8\% viennent à la ville. Entre 1830-40 la proportion n'est plus que

18 Par exemple le diocèse d'Orléans. Cf. Christiane Marcilhacy, Le diocèse d'Orléans au milieu du XIX $X^{e}$ siècle (Paris, Sirey, 1964), 218 et suivantes. Sur 188 prêtres ordonnés avant 1850 , $63 \%$ viennent de la ville. Un recrutement fortement urbain persiste à Nantes et à La Rochelle; Michel Lagrée, Mentalités, religion et histoire en Haute-Bretagne au XIX $X^{e}$ siècle. Le diocèse de Rennes 1815-48 (Paris, Klinsieck, 1977), 180-81. 


\section{c) L'origine sociale}

Nos connaissances sur l'origine sociale du clergé sont aussi sommaires que celles de l'origine géographique. Voilà pourquoi une sociographie des vocations s'avère aussi utile qu'une analyse de l'origine spatiale. Que savons-nous d'abord de la Nouvelle-France sur le sujet? Rappelons brièvement ce que nous a appris une étude de Pierre Hurtubise $^{19}$, pour le Régime français (1650-1762): sur 841 vocations sacerdotales et religieuses, l'historien a colligé des renseignements de type socio-professionnel pour les deux tiers des recrues. Regroupant les résultats sous deux rubriques: a) familles de notables (nobles, officiers, professionnels, marchands); b) familles du peuple (petits commerçants, artisans, manoeuvres, paysans ou habitants), Hurtubise attribue l'étiquette de populaire aux 300 familles pour lesquelles il n'a pu identifier la profession du père. Cette déduction est peut-être imprudente. Au reste, les catégories «notable» et "populaire» sont imprécises. Quelle que soit la pertinence du classement, les résultats des comptages en fonction de ces deux rubriques donnent la répartition suivante: a) familles de notables, $46,5 \%$; b) familles du peuple, $53,5 \%$. Pour les prêtres seulement, les proportions sont respectivement de $42 \%$ et $58 \%$. L'enquête pour ce dernier groupe aurait porté sur une liste de quelque 200 grands séminaristes. Même s'il s'agit d'un petit nombre pour une longue période, il faudrait pouvoir distinguer les recrues urbaines des recrues rurales, et surtout traiter avec prudence le XVII ${ }^{\mathrm{e}}$ siècle, époque où la société n'est pas encore parvenue à un état assez avancé de structuration. Quelle que soit la valeur de la statistique, retenons pour l'instant l'hypothèse d'une «fécondité relative» élevée de la notabilité.

Depuis le début des travaux sur le milieu d'origine du clergé français, le titre patrimonial est apparu comme la source la plus susceptible de révéler avec exactitude la profession du père de l'ordinand. Cet acte de rente, rédigé en faveur des ordinands au cours de leurs études théologiques, fournit des indications comparables au contrat de mariage ${ }^{20}$ lorsque les titreurs sont soit le père, soit la mère ou les deux parents du sujet. Qu'il y ait ou non lien de parenté avec le futur prêtre, le nom du titreur apparaît, ainsi que le lieu de son domicile, sa fonction socioéconomique, sa qualité, son statut ou quelque titre honorifique. Au Québec, le montant de la rente équivaut à $5 \%$ de la valeur du capital $(150 \# / 3000 \#)^{21}$, en principe un immeuble hypothéqué pour l'occa-

\footnotetext{
19 «L'origine sociale des vocations canadiennes de Nouvelle-France», Session d'étude 1978 de la Société canadienne d' histoire de l'Église catholique, 41-56.

20 Cf. André Viala, «Suggestions pour une histoire sociale du clergé aux temps modernes», dans Études d' histoire du droit canonique dédiées à Gabriel Le Bras (Paris, Sirey, 1965), 2: 1471 81. Pour plus de renseignements sur cette source, voir en particulier Yves-M. Le Pennec, «Le recrutement des prêtres dans le diocèse de Coutances au XVIII ${ }^{\mathrm{e}}$ siècle», Revue du département de la Manche (1970): 191-234.

21 Il y a des exceptions.
} 
sion, et dont la valeur est attestée par la présence de témoins. La rente est, sauf exception, viagère, c'est-à-dire non transmissible par voie d'héritage; certaines clauses restrictives stipulent parfois qu'elle est exigible seulement jusqu'à l'occupation d'une charge rémunérée, un bénéfice ecclésiastique permettant au prêtre de vivre de son travail. L'évêque ne prisait pas ce genre de restrictions ${ }^{22}$, qui fut progressivement abandonné. Comme la promesse de mariage, le titre sacerdotal est publié au prône, question de faire connaître la charge qui pèse désormais sur l'immeuble ${ }^{23}$.

Ces prémisses étant posées, il importe de connaître le rapport entre le nombre de titrés et le nombre d'ordinands. Mais au préalable, il faut estimer, ne fut-ce que grossièrement, le taux de conservation de la source dans les archives diocésaines. Pierre Hurtubise n'a retracé qu'un nombre insignifiant de titres pour tout le Régime français ${ }^{24}$. Beaucoup plus substantielle, notre série rassemble l'expédition des actes conservés aux archives diocésaines. Posons l'hypothèse qu'il s'agit d'un nombre sensiblement égal à celui des prêtres titrés. Présumons que les titres manquants sont également répartis dans le temps. Nous obtenons dès lors une distribution qui témoigne de fluctuations majeures du rapport ordinands/titrés.

La proportion de prêtres titrés $(70 \%)$ correspond à des pourcentages observés dans des études européennes ${ }^{25}$, ce qui accrédite l'hypo-

22 «J'ai vu le titre de Mr. Bégin, qui est à sa seconde publication. Il porte une clause que je n'aime pas parce qu'elle a déjà embarrassé et il serait à désirer que les notaires s'accoutumassent à l'omettre: c'est celle-ci; jusqu'à ce que le dit sieur $N$. soit pourvu d'un bénéfice. Il faudrait que la pension fut déclarée viagère sans cette restriction». Archives de l'Archevêché de Québec (AAQ), Registre des lettres, Plessis à Lartigue, 30 août 1821 .

${ }_{23}$ L'arrentement, comme le contrat de mariage, n'était pas universel. S'il était d'usage de rédiger un contrat de mariage pour les couples issus de familles à l'aise, il n'en allait pas de même du titre clérical. Celui-ci pouvait paraître d'autant plus nécessaire aux autorités diocésaines que l'ordinand provenait d'une famille sans ressource. La rente avait principalement pour but d'assurer la subsistance à un prêtre malade, même si le recours à la caisse d'assistance diocésaine pouvait, en cas de nécessité, assurer le revenu vital (Lucien Lemieux, «La première caisse ecclésiastique du clergé canadien», Session d'étude 1977 de la Société canadienne d'histoire de l'Église catholique, 5-22). Ce dispositif de secours favorisait la dispense de titre. De même une recrue en santé pouvait être élevée au diaconat au titre de service de l'Église ou encore au titre de mission; sans patrimoine, ni bénéfice assuré, le candidat offrait ses services au diocèse. Assuré de sa subsistance parce qu'il était à la charge de ses confrères advenant quelque maladie ou infirmité chronique, le sous-diacre pouvait donc être exempté de l'exigence canonique sans trop de formalités. Les motifs de dispense étaient jugés par l'évêque. Ce dernier était soumis à certaines directives romaines; à l'occasion, l'instance diocésaine recevait du pape une permission spéciale précisant le nombre d'exemptions pour une période déterminée. Dans l'ensemble des pays catholiques, la situation matérielle des parents et du futur prêtre, son état de santé, la «pureté» de sa vocation, la demande de pasteurs sont autant de motifs susceptibles de justifier une dispense de titre.

2416 pour 200 ordinations; Hurtubise, art. cit., 51-52.

25 Par exemple, M.-L. Fracart a estimé, dans une étude portant sur 368 prêtres, que $80 \%$ de ceux-ci étaient titrés, même si elle n'a repéré que 109 titres. Fracart, «Le recrutement du clergé séculier dans la région niortaise au XVIII ${ }^{\mathrm{e}}$ siècle», Revue d'histoire de l'Église de France, 159 (1971): 248-56. Dominique Julia a recensé 82\% de recrues titrées lors d'une étude portant sur 449 sujets du diocèse de Reims entre 1738 et 1773 . Julia, «Le clergé paroissial du diocèse de Reims à la fin du XVIII ${ }^{\mathrm{e}}$ siècle», Études ardennaises (avril-juin 1967): 22. 
TABLEAU 5

POURCENTAGE DE' PRÊTRES TITRÉS PAR RAPPORT AU NOMBRE D'ORDONNÉS SELON LA COLLECTION DE TITRES CONSERVÉS AUX ARCHIVES DIOCÉSAINES

(QUÉBEC 1775-1840)

\begin{tabular}{l|c|c|c}
\hline & & \multicolumn{2}{|c}{ Prêtres titrés } \\
Périodes & Ordinations & Nombre absolu & $\%$ \\
\hline $1775-80$ & 33 & 14 & 42 \\
$1781-86$ & 35 & 21 & 60 \\
$1787-92$ & 38 & 21 & 55 \\
$1793-98$ & 30 & 22 & 73 \\
$1799-04$ & 19 & 15 & 78 \\
$1805-10$ & 25 & 24 & 96 \\
$1811-16$ & 39 & 36 & 92 \\
$1817-22$ & 46 & 43 & 93 \\
$1823-28$ & 66 & 45 & 68 \\
$1829-34$ & 70 & 38 & 54 \\
$1835-40$ & 83 & 56 & 67 \\
\hline Total & 484 & 335 & $70 \%$ \\
\hline
\end{tabular}

thèse d'un taux de conservation de la source qui se rapproche du nombre de prêtres titrés. Si tant est que le nombre de titres conservés correspond à peu près au nombre de rentiers, il y a lieu de noter que vers le début du XIX ${ }^{\mathrm{e}}$ siècle la détention du titre clérical semble devenue une condition presque absolue pour recevoir la prêtrise. Pour la période $1805-$ $22,93 \%$ des prêtres sont bénéficiaires d'une rente, contre $60 \%$ pour les trente années qui précèdent, $63 \%$ pour les 18 dernières années. Compte tenu de l'ampleur des fluctuations, on peut présumer qu'à partir du moment où le recrutement urbain cède la place au recrutement rural, l'exigence canonique est appliquée dans toute sa rigueur. Cette précaution était peut-être jugée moins nécessaire à l'époque où les nouveaux prêtres étaient originaires de la ville épiscopale, ou venaient de l'agglomération montréalaise bien encadrée par les Sulpiciens. Les «familles à prêtres» et les candidats à la prêtrise des villes faisaient probablement l'objet d'une sollicitude particulière. Pouvait-on d'emblée faire confiance aux candidats venant du lointain plat pays? À la longue toutefois, la «crédibilité» de la campagne s'accroît tant et si bien que l'ordination sans titre redevient une pratique courante.

Quelque fiable qu'elle soit, la recherche de la profession paternelle via le titre clérical présente des difficultés qu'on ne doit pas sous-estimer. Si les titreurs étaient communément les père et mère des ordinands, la tâche serait relativement facilitée. Tel n'est pas le cas, comme 
TABLEAU 6

PROFESSIONS DES FAMILLES DE PRÊTRES D'APRÈS LES TITRES CLÉRICAUX ET AUTRES SOURCES D'APPOINT

(QUÉBEC 1775-1840) ${ }^{1}$

\begin{tabular}{|c|c|c|c|c|c|c|c|c|c|c|c|c|c|c|c|}
\hline \multirow[b]{2}{*}{ Années } & \multicolumn{6}{|c|}{ Recrues urbaines } & \multicolumn{6}{|c|}{ Recrues rurales } & \multirow[b]{2}{*}{ 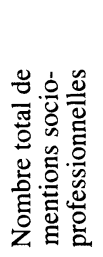 } & \multirow[b]{2}{*}{ 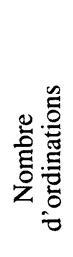 } & \multirow[b]{2}{*}{ 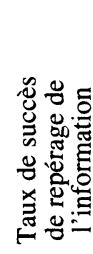 } \\
\hline & 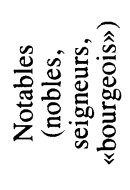 & 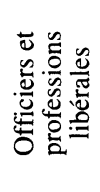 & 㟒 & 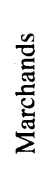 & 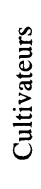 & $\stackrel{\mathscr{E}}{\stackrel{\Xi}{2}}$ & $\begin{array}{l}\frac{\mathscr{0}}{0} \\
\frac{\pi}{0} \\
z\end{array}$ & 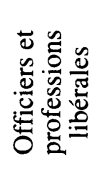 & 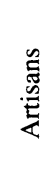 & 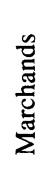 & 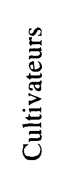 & 总 & & & \\
\hline $\begin{array}{l}1775-1780 \\
1781-1786 \\
1787-1792 \\
1793-1798 \\
1799-1804 \\
1805-1810 \\
1811-1816 \\
1817-1822 \\
1823-1828 \\
1829-1834 \\
1835-1840\end{array}$ & $\begin{array}{l}1 \\
2\end{array}$ & $\begin{array}{l}1 \\
2 \\
1\end{array}$ & $\begin{array}{l}2 \\
6 \\
5 \\
4 \\
3 \\
5 \\
5 \\
4 \\
2 \\
5 \\
1\end{array}$ & $\begin{array}{l}2 \\
1 \\
4 \\
3 \\
1 \\
2 \\
2 \\
\\
3 \\
1\end{array}$ & 1 & $\begin{array}{l}1^{3} \\
2^{5} \\
1^{6} \\
1^{9}\end{array}$ & $\begin{array}{l}1 \\
2\end{array}$ & $\begin{array}{l}1 \\
2 \\
1\end{array}$ & $\begin{array}{l}3 \\
3 \\
2 \\
2 \\
4\end{array}$ & $\begin{array}{l}1 \\
1 \\
1 \\
1 \\
1 \\
2 \\
1\end{array}$ & $\begin{array}{c}1 \\
4 \\
2 \\
5 \\
10 \\
12 \\
12^{10} \\
10^{11} \\
21\end{array}$ & $\begin{array}{l}1^{2} \\
1^{4} \\
1^{2} \\
3^{8} \\
1^{4} \\
1^{4} \\
5^{7,4} \\
1^{4}\end{array}$ & $\begin{array}{r}8 \\
11 \\
17 \\
15 \\
7 \\
16 \\
23 \\
24 \\
23 \\
29 \\
31\end{array}$ & $\begin{array}{l}33 \\
35 \\
38 \\
30 \\
19 \\
25 \\
39 \\
46 \\
66 \\
70 \\
83\end{array}$ & $\begin{array}{l}25 \% \\
30 \% \\
45 \% \\
50 \% \\
37 \% \\
64 \% \\
59 \% \\
52 \% \\
35 \% \\
41 \% \\
37 \%\end{array}$ \\
\hline Total & 6 & 7 & 42 & 19 & 1 & 5 & 5 & 5 & 15 & 8 & 77 & 14 & 204 & 484 & $43 \%$ \\
\hline
\end{tabular}

1. Nous reprenons l'intitulé ainsi que la classification, légèrement amendée, de Timothy Tackett dans «L'histoire sociale du clergé

6. Capitaine et propriétaire de goélette. diocésain dans la France du XVIII ${ }^{\mathrm{e}}$ siècle», Revue d'histoire moderne et contemporaine (1979): 227.

$$
\text { 2. Navigateur. }
$$$$
\text { 7. Sacristain }
$$

8. Un navigateur, 2 anciens capitaines de milice. 9. Écuyer.

4. Officier de milice.

10. Dont un cultivateur maître-charpentier.

11. Dont un cultivateur maître-forgeron.

5. Un juge de paix; un veuf devenu prêtre dont le fils devient aussi prêtre 
en témoignent les études menées sur ce genre de source ${ }^{26}$. La pauvreté des parents, le poids des charges familiales, la donation entre vifs survenue avant l'entrée du candidat aux études théologiques, le décès des parents, sont autant de facteurs qui expliquent pourquoi les titreurs ne sont pas toujours, loin de là, le père et la mère du futur prêtre. Quand des frères, des oncles, des grands-parents, des cousins, et plus encore quand des protecteurs sans lien de parenté avec l'ordinand hypothèquent un bien pour soutenir une vocation, la mention des nom, prénom et profession du père n'apparaît que très exceptionnellement dans l'acte de rente. Entre 1775 et 1840, les parents des prêtres nés au Québec ont été les titreurs de leur fils pour près de $60 \%$ des bénéficiaires. Aussi bien, le renseignement sur l'origine sociale reporté au tableau suivant provient principalement de cette source. On y a ajouté une trentaine de mentions socio-professionnelles provenant soit de monographies de paroisses, soit du Dictionnaire biographique du Canada, soit enfin des actes de baptềmes ${ }^{27}$.

L'origine géographique étant connue pour 399 des 484 ordinands, le pourcentage des mentions socio-professionnelles est légèrement plus élevé pour les recrues urbaines, $46 \%$ (80/172), que pour les vocations rurales, 54\% (124/227). L'échantillon forcé qui sert de base à la sociographie des vocations est sensiblement plus mince que celui sur lequel s'appuyait la géographie du recrutement. Quand on sait que certaine étude européenne a été menée à l'aide d'un repérage de l'information représentant quelque $10 \%$ du groupe à l'étude ${ }^{28}$, on se sent pour ainsi dire rassurés sur la représentativité des séries qui fournissent une désignation occupationnelle ou autre pour plus de 40\% (204/484) des ordinands. Par contre, la cueillette de l'information s'est avérée très inégale selon les périodes; avec un échantillon forcé de 25-30\% pour la première décennie, la prudence est de règle. L'échantillon couvre mieux

\footnotetext{
$26 \quad$ Voici quelques exemples: pour Coutances, Yves Le Pennec a retrouvé 300 mentions socio-professionnelles dans 1300 actes d'arrentement. Le Pennec, «Le recrutement des prêtres dans le diocèse de Coutances au XVIII ${ }^{\mathrm{e}}$ siècle», Revue du département de la Manche (1970): 201. L'étude du diocèse de Lisieux au XVIII' siècle a donné les résultats suivants: pour 1404 ordinands, 997 titres ont été constitués par les familles, ce qui comprend non seulement les pères et les mères, mais aussi les grands-parents, les frères, oncles et cousins; 407 titreurs, près de $30 \%$, sont des «bienfaiteurs». Charles Berthelot du Chesnay, «Le clergé diocésain français au XVIII siècle et les registres des insinuations ecclésiastiques", Revue d'histoire moderne et contemporaine (1963): 256. Pour le diocèse de Rennes, Michel Lagrée a rassemblé 93 titres constitués entre 1803-10. Dans 22 cas, le capital de la rente provient du futur prêtre. Plus de $50 \%$ des candidats rennais ont un titre garanti par des biens de famille, quel que soit le lien de parenté; 19 individus ont recours à des étrangers pour s'assurer la rente prescrite. L'exemple rennais est ainsi commenté par Lagrée: «C'est surtout chez les paysans que la famille est mise à contribution: les parents affectent à cet effet une ou plusieurs pièces de terre, des frères et soeurs acceptent de sacrifier une part de l'héritage... Quant à ceux qui ne trouvaient pas chez eux les 300 francs, il leur fallait trouver un riche protecteur, rôle dévolu parfois à des ecclésiastiques... mais aussi à des laïcs». Lagrée, op. cit., 18-81.

Le coup de sonde dans les actes de baptêmes s'est avéré peu fructueux. De plus, comme un quart de siècle s'écoule entre la naissance et la prêtrise, présumer qu'il n'y a pas eu mobilité sociale du père est imprudent. Voilà pourquoi nous avons abandonné la consultation de la source.

28 C. Marcilhacy, op. cit., 222.
} 
la réalité pour les décennies durant lesquelles le recrutement rural est en hausse, quoique l'information soit plus clairsemée pour les quinze dernières années. En faisant abstraction du début et de la fin de la série, on peut dire que la représentativité de l'échantillon paraît suffisante. Le nombre élevé de titrés ruraux ne saurait surprendre. Ainsi que l'a noté Michel Lagrée pour la Bretagne, c'est surtout chez les familles paysannes que le patrimoine est mis à contribution ${ }^{29}$.

Si elle est un bon indicateur de l'identité sociale du père, il faut néanmoins admettre que l'étiquette socio-professionnelle est une donnée imprécise; elle ne révèle pas, tant s'en faut, le statut économique des familles en cause ${ }^{30}$. Ces réserves faites, quelles observations se dégagent du tableau?

Recrues rurales et urbaines confondues, il faut noter le faible apport des grandes familles seigneuriales et aristocratiques, si l'on soustrait du groupe de «notables» l'unique «bourgeois» recensé sous cette rubrique. Eu égard aux effectifs globaux de la population active, le groupe des artisans urbains est probablement sur-représenté. Il en est de même des marchands quoiqu'à un degré présumément moindre. En revanche, il semble que les élites de la culture savante, fonctionnaires, professionnels du droit et de la médecine ne sont pas pourvoyeuses de prêtres. Encore faut-il noter la «fécondité différentielle» entre sous-groupes. Pour deux fils du même avocat et juge, il se trouve cinq enfants de notaires. Fait à souligner: parmi les frères de prêtres qui ont constitué le titre clérical, on compte quatre notaires contre aucun avocat ou médecin.

À la campagne, le nombre de cultivateurs ne saurait surprendre. Il faut plutôt encore ici noter la proportion relativement élevée d'artisans. Il se trouve, comme à la ville, deux fois moins de fils de marchands que de descendants d'artisans. Dans ce sous-groupe, à la ville comme à la campagne, les pères de prêtres exerçant un métier lucratif comme celui de forgeron, d'orfèvre ou de tonnelier ${ }^{31}$ représentent un nombre considérable par rapport à l'ensemble des artisans. Refoulés dans la catégorie autre, les capitaines de milice sont présumément de gros cultivateurs. Le groupe paysan paraît suffisamment à l'aise, puisqu'il est en mesure de supporter un fils qui devient prêtre. L'ensemble de ces indices suggère que le clergé de notre ancien régime finissant provient en bonne partie des «classes moyennes». Un plus petit nombre serait originaire de milieux familiaux moins à l'aise. Faut-il rappeler que $17 \%$ (55) des titreurs sont des protecteurs sans lien déclaré de parenté avec le futur

\footnotetext{
29 M. Lagrée, op. cit., 181.

30 «Les dénominations socio-professionnelles doivent être soigneusement distinguées du statut éonomique. Une analyse complète des origines familiales du clergé devrait prendre en considération les niveaux de fortune.» T. Tackett, art. cit., 215.

31 La même observation a été faite pour la France du XVIII ${ }^{\mathrm{e}}$ siècle. Tackett, art. cit., 215
} 
prêtre? Parmi les recrues de familles pauvres, il faut peut-être compter ces quelques orphelins qu'un coup de sonde a permis d'identifier. Ces recrues sont prises en charge par un prêtre. Parmi les titreurs-bienfaiteurs, dix sont des prêtres. Ainsi donc des curés fortunés peuvent payer les études secondaires et le cas échéant, titrer un ordinand orphelin de père.

S'il est délicat de pressentir le niveau de fortune des parents, leur niveau culturel est moins difficile à identifier. Quelques marchands ont peut-être fait une partie de leurs études collégiales dans l'un ou l'autre des petits séminaires provinciaux. Artisans et paysans n'ont pas, sauf exception, subi l'influence de la culture savante autrement que par la fréquentation de l'église, de la petite école, ou encore la rencontre de professionnels du droit ou de la médecine. Un certain nombre a lu ou entendu lire un journal. Pour les prêtres issus de ces familles, le niveau culturel d'arrivée n'est par conséquent pas comparable au niveau culturel à l'entrée au petit séminaire. Le passage de la culture "première» à la culture «seconde» atteint les fils de cultivateurs comme les gens de métier. Les enfants de familles de fonctionnaires, ceux dont le père exerce une profession libérale ne vivraient pas, se faisant prêtres, une métamorphose culturelle comparable à celle du petit paysan. Or, c'est dans ces groupes instruits que les vocations se font rarissimes, sauf peut-être au sein de la profession notariale. Le haut niveau d'instruction des parents serait-il un obstacle à l'option sacerdotale?

\section{II- La mise en contexte du prélèvement statistique}

La mutation qui s'effectue entre la fin du XVIII' siècle et les premières décennies du XIX était jusqu'ici inconnue. D'entrée de jeu, il faut pourtant reconnaître que le fait n'est pas isolé. Quiconque a parcouru l'historiographie religieuse des diocèses de France des vingt-cinq dernières années ne se surprend pas d'observer un trend laurentien analogue aux mutations d'outremer, elles aussi caractérisées par un déplacement de l'origine spatiale vers les zones rurales, et par la croissance marquée des familles paysannes de modeste aisance comme pourvoyeuses de prêtres ${ }^{32}$. Le changement qui se produit des deux côtés de l'Atlantique est plus facile à identifier qu'à expliquer. Faut-il reconnâ̂tre, avec deux ou trois décennies de «retard» pour le Québec, un cheminement très proche de la trajectoire française? La philosophie des lumières a-t-elle miné, ici, comme là-bas, le prestige rattaché à la prêtrise, au sein des minorités sociales frottées à la culture savante? Ce facteur a probablement joué son rôle, comme en témoignent les études sur l'influence du rationalisme et du libéralisme sur les classes moyennes

32 Pour l'ensemble de la France au dernier tiers de l'Ancien régime, Tackett note une «ruralisation relative... popularisation des origines sociales... désertion de plus en plus nette des élites urbaines». Tackett, art. cit., 217. 
canadiennes-françaises ${ }^{33}$. Mais on ne peut présumer, du moins dans l'état actuel des connaissances, une quelconque déchristianisation populaire, responsable en France d'une baisse de la demande sacerdotale. En revanche, il est probable que la présence protestante, massive dans les villes vers 1830-40, ait détourné, ici comme là-bas, des jeunes de la prêtrise ${ }^{34}$.

La croissance des candidats d'extraction paysanne représente une structure de remplacement à la défection des élites urbaines de la culture et de l'argent. Comme le démontrent les études françaises, elle caractérise le XIX ${ }^{\mathrm{e}}$ siècle. Dans le diocèse d'Arras, étudié pour les décennies antérieures à la guerre de 1914, on a expliqué la prédominance rurale des ordinands par l'activité promotionnelle de prêtres responsables d'écoles latines, par l'implantation de collèges ruraux et aussi par la présence de tensions démographiques réduisant les perspectives d'établissement et incitant à la prêtrise ${ }^{35}$. L'un ou l'autre de ces facteurs a joué un rôle dans la campagne québécoise dès les premières décennies $\mathrm{du} \mathrm{XIX}^{\mathrm{e}}$ siècle.

Dans l'état actuel des connaissances, il demeure difficile de préciser à partir de quand s'est structuré, au Québec, le modèle urbain de recrutement que nous observons encore à la fin du XVIII ${ }^{e}$ siècle. Pour la France, on sait que la volonté de réforme a pris naissance dans les villes après le concile de Trente, puis a progressivement gagné les campagnes. Timothy Tackett observe cette orientation dans les diocèses d'Autun et de Toulouse où «la proportion des fils de citadins et de notables entrant dans le clergé atteint son apogée dans les dernières décennies du XVII ${ }^{\mathrm{e}}$ siècle... avant l'introduction des séminaires et la mise en application plus stricte de la règle du titre patrimonial... le clergé a été plus populaire dans ses origines sociales ${ }^{36}$. Des séries chronologiques combinant à la fois l'origine sociale et géographique des recrues de la Nouvelle-France pourraient peut-être montrer les similitudes ou les contrastes avec l'itinéraire français, si toutefois les nombres ne sont pas trop petits pour autoriser certaines conclusions. Quoi qu'il en soit, ce n'est pas la voie empruntée par Hurtubise qui a regroupé les vocations religieuses et sacerdotales sans se soucier de la répartition rurale/urbaine. Aurait-il voulu suivre la voie tracée par les études européennes qu'il se serait buté à d'autres difficultés peut-être insurmontables. En grande majorité, les prêtres viennent de France. Encore au milieu du XVIII ${ }^{e}$ siècle, la moitié des réguliers et séculiers réunis sont

\footnotetext{
33 L'étude pionnière de Marcel Trudel, L'influence de Voltaire au Canada (Montréal, Fides, 1945, 2 v.), a été suivie comme on sait de nombreux articles de Fernand Ouellet, qui exhumaient l'influence "philosophique» longtemps méconnue.

34 Les facteurs d'explication avancés ici pour l'exemple français sont tirés de Tackett, art. cit., 202, 206-08.

${ }_{35}$ Y.-M. Hilaire, Une chrétienté au XIX $X^{e}$ siècle? La population du diocèse d'Arras (18401914) (Lille, Presses de l'Université de Lille III, 1977), I: 292-95.

36 Tackett, art. cit., 213 et 217.
} 
des Français. À compter des dernières décennies du XVIIIe siècle, par contre, le recrutement est largement autochtone. C'est seulement à la suite de la Révolution qu'un notable détachement de prêtres originaires de France vient se joindre aux «pasteurs» québécois.

En l'absence de renseignements plus précis sur la période française, il faut, semble-t-il, continuer de considérer comme valide l'hypothèse de Louise Dechêne. L'Église de la Nouvelle-France, écrit-elle, «a toujours misé uniquement sur les classes supérieures pour son recrutement». La persévérance scolaire des petits séminaristes est plus élevée «dans les groupes urbains intermédiaires, hôteliers, boulangers, notaires, chirurgiens, etc. qui valorisent l'instruction mais ont rarement de grands moyens... Plus d'un siècle va s'écouler avant que l'Église songe à utiliser des paysans pour encadrer la population des côtes $»^{37}$. Pour étayer la thèse de l'origine «populaire» des vocations de la Nouvelle-France, Hurtubise rappelle qu'au petit séminaire de la ville épiscopale, un système de bourses d'études vient compenser le manque de ressources des parents. C'est là un point de vue partagé jadis par les historiens de l'Église soucieux de préserver l'image de l'accessibilité aux études sans égard au niveau de fortune des parents ${ }^{38}$. Or vers 1740 , il en coûte 250 livres pour loger et nourrir un fils au séminaire de Québec. Parmi ceux qui n'habitaient pas la ville, combien de parents ont reçu une remise partielle ou totale sur le prix de la pension? Faute d'étude comptable, il faut pour l'instant rester coi devant la discordance des témoignages. Louise Dechêne cite l'exemple d'une veuve qui obtient difficilement pour son fils une bourse d'études du séminaire. À ce témoignage du début du XVIII ${ }^{\mathrm{e}}$ siècle, il faut ajouter celui de l'évêque lui-même. En 1733, il impute le petit nombre de vocations canadiennes à l'incapacité où se trouvent les parents de défrayer les coûts de la pension $^{39}$.

À l'orée du XIX ${ }^{\mathrm{e}}$ siècle, une nouvelle stratégie de recrutement est en voie de structuration. C'est l'époque où les clercs observent le tarissement des couches supérieures et moyennes de Québec et de Montréal. Quels que soient les motifs d'une nouvelle demande cléricale dirigée vers la paysannerie, l'Église implante de petits séminaires en région rurale. Jusqu'en 1803, deux collèges seulement, l'un à Québec, l'autre à Montréal, constituaient les seules voies d'accès normal à la prêtrise. Un troisième petit séminaire est fondé à la campagne, dans le seul district qui n'en était pas jusque-là pourvu. On ne l'établit pas dans la petite ville de Trois-Rivières, mais dans un village de la rive sud du Saint-Laurent: Nicolet. La région administrative centrale du Québec est

\footnotetext{
37 Habitants et marchands de Montréal au XVII siècle (Paris, Plon, 1974), 477s.

38 Voir à ce propos Amédée Gosselin, L'instruction au Canada sous le régime français (1635-1760) (Québec, Laflamme, 1911), passim. note 18 .

Cité par Louis-E. Hamelin, art. cit., Recherches sociographiques (avril-juin 1961): 196,
} 
alors beaucoup moins peuplée que les districts adjacents. L'évêque espère faire de Nicolet cette pépinière de vocations devenue nécessaire pour assurer la relève. Le vieillissement du clergé en exercice n'est-il pas alarmant? Entre 1790 et 1805 le nombre de prêtres de plus de 35 ans est passé de $58 \%$ à plus de $73 \%$. Il culmine à $80 \%$ en 1810 . C'est seulement vers 1840 que la répartition par groupe d'âge donnera une ventilation identique à celle de $1790^{40}$.

$\mathrm{Au}$ cours du premier demi-siècle d'existence, la presque totalité des collégiens de Nicolet viennent de la campagne, la plupart des paroisses environnantes ${ }^{41}$. Il en est de même pour le second collège fondé en 1827-29 dans le district de Québec, Sainte-Anne-de-la-Pocatière, à quelque 100 kilomètres en aval de Québec ${ }^{42}$. En plus des nouveaux collèges, des curés de campagne tiennent des écoles presbytérales où l'on enseigne le latin pour préparer des jeunes à l'entrée au petit séminaire. On en compte une à l'Ange-Gardien de 1797 à 1805, une autre inaugurée un peu plus tard par le curé de Saint-Pierre-deMontmagny. Dans le district de Montréal, ces écoles préparatoires ou encore de véritables collèges apparaissent aussi au cours des années 1810-30. À Boucherville, à Laprairie, puis à Sainte-Thérèse-de-Blainville (embryon du futur collège), des classes latines sont tenues par le prêtre paroissial, cependant que les curés de Chambly, de Saint-Denissur-Richelieu fondent des collèges. Mais ces deux institutions collégiales firent long feu, contrairement au collège de Saint-Hyacinthe inauguré au début des années $1810^{43}$. Plusieurs prêtres issus des couches de la paysannerie choisiront le sacerdoce grâce à cette institution implantée dans une région à forte croissance démographique.

Si la demande de prêtres auprès des humbles foyers du plat pays s'est montrée aussi ferme, c'est que le clergé n'était pas sans observer le détachement des élites urbaines, et surtout la tiédeur qui gagnait la petite bourgeoisie, milieu désormais peu enclin à la «sacerdotalisation» de ses fils. Au collège de Montréal, au cours des premières décennies du XIX ${ }^{\mathrm{e}}$ siècle, il se trouve, année moyenne, seulement trois ou quatre finissants qui choisissent le sacerdoce ${ }^{44}$. L'évêque confie en 1807: «Les Canadiens qui jouissent de quelque considération dans la province» sont plus sensibles aux «espérances» du siècle qu'aux «intérêts de Dieu». Les hommes de loi s'éloignent, pense-t-il, de l'Église à cause de sa position sur le prêt à intérêt, «de sorte qu'il y a très peu d'avocats et de

\footnotetext{
40 Calculs effectués à partir des tableaux de James Lambert, op. cit., Appendix 1, «Age Structure of the Clergy, 1660-1870», 665 1971): 87

42 Ulric Lévesque, «Les élèves du collège de Sainte-Anne-de-la-Pocatière (1829-1852)», RHAF, XXI, 4 (mars 1968): 774-791.

43 Claude Galarneau, Les collèges classiques au Canada-français (1620-1970) (Montréal, Fides, 1978), 16-24.

44 Louis Rousseau, La prédication à Montréal 1800-1830 (Montréal, Fides, 1977), 43.
} 
notaires qui fréquentent les sacrements, et que plusieurs s'élèvent contre le clergé comme maintenant une doctrine pernicieuse et nuisible au commerce et à l'intérêt public»; Plessis oppose à ces frondeurs de la culture savante, émancipés de la tutelle cléricale, la masse paysanne de bons chrétiens, jugée ignorante et sans influence, mais pieuse, zélée et respectueuse des «choses saintes» ${ }^{45}$. Comme l'épiscopat français le déplorait trente ans plus tôt, la philosophie rationaliste accouchait ici de notables peu enclins aux attitudes onctueuses vis-à-vis des hommes de Dieu. Ne pouvant compter sur l'appui des professionnels pour défendre le statut de l'Église, l'évêque mise sur les couches populaires urbaines et les familles d'artisans pour produire des prêtres ${ }^{46}$. C'est de ce milieu et de la paysannerie qu'il compte tirer la relève, assuré que notaires, avocats, médecins, anciens de collèges donneront peu ou prou de fils à l'Église. Les prêtres font du reste l'objet d'attaques nourries de la part de la petite bourgeoisie abreuvée, comme le théologien, à la culture savante, mais dont le contenu livresque est plutôt voltairien ou simplement «encyclopédique». Après la mort de Plessis, des législations «Votées» par la petite bourgeoisie montrent les progrès du laïcisme. À la fin des années 1820, la loi des écoles de l'Assemblée législative, puis le projet de démocratisation des fabriques cristallisent l'opposition de la petite bourgeoisie instruite au curé de campagne ${ }^{47}$. Un certain laïcisme est à l'origine de projets d'institutions collégiales aussitôt mis en échec par les initiatives du clergé.

Si l'opposition au prestige, au pouvoir du prêtre a eu des répercussions dans les rapports Église-État, c'est qu'il existe bel et bien dans les familles bourgeoises un préjugé défavorable à l'endroit de la prêtrise. Observons le destin d'Édouard-Charles Fabre, le futur archevêque de Montréal, fils du libraire et imprimeur Édouard-Raymond. Idéologiquement commis à la cause des rebelles de 1837 , le père est déçu de ce que le fils lui écrive depuis Paris son désir de choisir la carrière sacerdotale: «tu me coûtes joliment de l'argent à Paris... je ne t'ai pas envoyé là pour t'occuper de ta vocation.» ${ }^{48}$ Entêté à devenir prêtre malgré les souhaits du père, Édouard-Charles aura gain de cause; mais pour une persévérance en dépit des desseins de la famille, peutêtre 10 , peut-être 20 «vocations manquées»! La demande sacerdotale en l'occurrence s'adresse donc au groupe le plus susceptible d'offrir des jeunes à l'Église: la paysannerie, ou plus précisément les élites

\footnotetext{
45 AAQ-Registre des lettres Plessis à Bourret, French Chapel, King's Street, London, lettre de mai 1807.

46 À la fin des années 1810, Plessis autorise l'ouverture d'un collège dans la paroisse de Saint-Roch de Québec, «où des jeunes gens, doués de talent et appelés à l'état ecclésiastique, pourraient à peu de frais commencer un cours d'études qu'ils iraient terminer soit au Séminaire de Québec, soit au Collège de Nicolet». Cité par Claude Galarneau, op. cit., 22.

47 Richard Chabot, Le curé de campagne et la contestation locale au Québec de 1791 aux troubles de 1837-38 (Montréal, Hurtubise-HMH, 1975).

${ }_{48}$ Cité par Jean-Louis Roy, Édouard-Raymond Fabre libraire et patriote canadien (17991954) (Montréal, Hurtubise-HMH, 1974), 29.
} 
rurales de l'agriculture et de l'artisanat. Conscient de la fécondité sociale différentielle des vocations, un prêtre déplore au début des années 1820 «le peu de vocations ecclésiastiques», qu'il explique en ces termes: «la presque totalité des jeunes gens doués de talent, ou appartenant à nos meilleures familles quoique nés avec des inclinaisons heureuses, dégoûtés du peu de respectabilité de notre corps, s'éloignent avec dédain du sanctuaire, et vont grossir tôt ou tard le nombre de nos adversaires.» ${ }^{49}$ Un promoteur laïque du collège de Chambly est plus explicite encore. Vers les mêmes années, il écrit au fondateur du collège de Saint-Hyacinthe:

Nos collèges des villes de Québec et de Montréal ne nous procureront jamais un nombre d'ecclésiastiques proportionné à celui que nous devons attendre de nos collèges de campagne. Les écoliers des villes en faisant leurs études, sont en général entretenus des projets de leurs parents, qui les destinent à devenir des notaires, des avocats, des membres de la législature et quelques-uns des ecclésiastiques; mais indifférents pour ce dernier état, et quand il faut se décider ils choisissent naturellement ceux dont les dehors et la liberté les flattent davantage. Il n'en est pas ainsi pour les écoliers de nos collèges de campagne; appartenant à des familles dont les vues les plus ambitieuses se portent à voir leurs enfants prêtres, ce dont leurs parents les entretiennent et les flattent en toute occasion, ils mènent dans leurs familles une vie uniforme et tranquille, avant d'aller au collège, et pendant le temps de leurs vacances, de sorte qu'ils désirent retourner à leurs études; ce qui n'est pas le cas avec les écoliers de nos villes et ces jeunes gens se disposent par degré à prendre un état qui leur paraît d'autant plus avantageux qu'ils ne peuvent désirer ceux dont l'éclat leur est inconnu. ${ }^{50}$

Admirable lecture de la conjoncture religieuse. Elle permet de comprendre la mise en place d'un nouveau «dispositif» de recrutement: des prêtres d'âge mûr, parvenus à la tête de paroisses lucratives, ont des revenus suffisants pour fonder des collèges avec l'aide des paroissiens, corvéables pour l'occasion, et aussi parfois de modestes allocations gouvernementales. Pour d'autres, il suffit de protéger, de patronner certains jeunes paroissiens avec l'accord des parents. Pour d'aucuns, la promotion sociale d'un fils doué, pieux est assurée. Les familles peuvent même y entrevoir des perspectives d'enrichissement; un membre de la famille devenu prêtre assurera un jour l'aide matérielle aux moins fortunés. Pour un curé bienfaiteur, l'aisance provient d'un prélèvement sur la récolte; attribuer une ou plusieurs bourses d'études, c'est investir en vue de la reproduction, prévoir l'encadrement une fois qu'il sera retiré ou décédé; le financement de l'affaire provient de ceuxlà mêmes qui réclament des prêtres encore trop peu nombreux pour

49 Cité par James Lambert, op. cit., 512.

50 Loc. cit. 
desservir en permanence toutes les paroisses de la campagne ${ }^{51}$. Accéléré par la création de collèges de campagne, le passage du recrutement urbain au recrutement rural a de multiples conséquences sur l'évolution de la société globale.

Lorsqu'au cours des années 1840 , le clergé prend l'initiative du mouvement de colonisation, il obéit à une impulsion profonde. Ce sont des frères et des soeurs qui sont à l'étroit sur le domaine familial des basses terres du Saint-Laurent. Le missionnaire qui accompagne les colons a des affinités avec les pionniers des nouvelles zones d'occupation du sol. Ce personnage un peu raboteux, à la voix tonitruante, n'a pas les raffinements de son homologue social du début du siècle. Ce nouveau curé de «frontière» est issu des rangs du petit peuple des campagnes. La lecture de sa correspondance laisse deviner une personnalité bon-enfant, un tantinet populiste, au langage coloré, aux manières un peu rustres, mais dont la colère dominicale ou les rigueurs plus discrètes du confessionnal ne scandalisent que les oreilles d'anciens collégiens $^{52}$. Observateur critique des moeurs cléricales, Louis-Antoine Dessaulles écrivait vers 1880: «Les curés sont devenus plus fanatiques à mesure qu'ils se sont recrutés davantage chez les paysans. Leur niveau social - j'entends ici le savoir-vivre et les manières distinguées était bien supérieur, il y a quarante ans à ce qu'il est aujourd'hui.» ${ }^{53}$ Un état du clergé en 1810 fait voir la distance parcourue entre l'époque où Dessaulles consigne ses réflexions et celle qu'il interpelle dans le souvenir à deux ou trois décennies près. Le clergé rural de 1810 compte 125 prêtres. Cette coupe exclut les prêtres des agglomérations de Québec, de Montréal et de Trois-Rivières, de même que les deux ou trois clercs affectés à l'enseignement à Nicolet. Par leurs origines, la majorité des curés de campagne ont peu d'affinités avec le milieu qu'ils desservent: deux sur trois sont originaires des villes de Québec et de Montréal $^{54}$. Une douzaine de ces prêtres sont des fils de marchands, de négociants, de «bourgeois», un nombre à peu près égal est issu du

\footnotetext{
51 Voici une statistique du début des années 30 , période déjà moins pauvre en ressources humaines que les premières années du siècle: il y a alors 183 paroisses, mais seulement 160 curés et 42 vicaires impartis aux titulaires des grandes cures. Archives de la Propangada Fide Scr. rif. VI, 1-10 Signay à Rome, 9 novembre 1832. Référence tirée du Fichier documentaire de Lucien Lemieux.

${ }_{52}$ On attend toujours une thèse sur ce curé de frontière du milieu du siècle, évoqué par Jean Hamelin et Yves Roby dans leur Histoire économique du Québec (Montréal, Fides, 1971), 168172. En attendant, on lira avec profit le chapitre IX de Normand Séguin, La conquête du sol au $X I X^{e}$ siècle (Montréal, Boréal Express, 1977).

53 Cité par Jean-Paul Bernard, «Les fonctions intellectuelles de Saint-Hyacinthe à la veille de la Confédération», Rapport 1980 de la Société canadienne d' histoire de l'Église, 17. La même lettre est citée par Pierre Savard dans Aspects du catholicisme canadien-français au XIX ${ }^{e}$ siècle (Montréal, Fides, 1980), 28. La lettre de Dessaulles à Paul Théodore-Vibert a été publiée dans le livre de celui-ci: La Nouvelle-France catholique. Une page d'histoire contemporaine dans le Nouveau-Monde. Le Canada clérical... (Paris, Librairie Schleicher Frères, 1908), 475-80.

54 Nos calculs sont faits d'après la liste nominative de l'Almanach de Québec et les renseignements biographiques tirés de Allaire et Tanguay.
} 
monde de l'artisanat (trois fils de maçons, deux d'orfèvres, trois de tonneliers, etc.); il se trouve trois fils de navigateurs, cinq de chirurgiens, trois d'hommes de loi, deux de seigneurs). Trente ans plus tard, la campagne québécoise est autosuffisante en matière de personnel ecclésiastique. C'est de son sein que proviennent ses curés.

En France, on fait remonter la légende des origines paysannes au livre de Pierre de Vaissière: Les curés de campagne de l'ancienneFrance $(1933)^{55}$. Au Québec, il semblerait qu'il en faille attribuer la paternité à Joseph-Edmond Roy. En 1904, l'historien écrit au tome quatre de son Histoire de la Seigneurie de Lauzon: "Ces curés d'autrefois... [début du XIX ${ }^{\mathrm{e}}$ siècle] aident d'autant plus les habitants à supporter leur sort, qu'il y a entre eux communauté d'origine et de pensées. Alors, comme aujourd'hui, les prêtres de la campagne sortaient en effet pour la plus grande partie du peuple et se recrutaient parmi les laboureurs.» ${ }^{56}$ On n'a cessé depuis lors de reprendre la thèse proposée, soutenue dans un passage où il était spécifiquement question des années 1800. Dessaulles n'aurait pas pu ainsi «pérenniser» rétrospectivement un milieu d'origine dont il connaissait l'historicité. Au début du XX siècle, le présent a été subrepticement assimilé au passé, parce que l'origine urbaine des clercs était déjà hors de portée de la mémoire «orale». En 1967, dans un article-synthèse sur le clergé du XIX ${ }^{\mathrm{e}}$ siècle, Pierre Savard a repris l'énoncé de l'historien de Lauzon ${ }^{57}$. Mais suivant les lois du genre, l'article de Savard ne s'appuyait pas sur une recherche originale. Il rééditait un énoncé maintes fois répété. Quant à LouisEdmond Hamelin qui, au cours des années 1960, publiait son étude sur l'évolution numérique du clergé canadien-français de 1633 à nos jours, il n'a pas posé la question de la fécondité respective de la ville et de la campagne; il a seulement énoncé un commentaire impressionniste accréditant l'hypothèse de l'origine rurale, sans se demander si cette structure de recrutement avait aussi caractérisé l'époque coloniale française. De fait, cette généralisation demeure valable pour une longue période s'amorçant au cours des années 1810-20. L'économiste-géographe a-t-il été induit en erreur par ses sources? Le tome 1 du Dictionnaire biographique du clergé de Allaire donne très exceptionnellement la mention de l'occupation du père. Or le renseignement socio-profes-

\footnotetext{
55 «Le clergé paroissial n'est donc pas d'abord rural, comme on l'a si souvent prétendu, à la suite notamment de Pierre de Vaissière». Dominique Julia, «Le clergé paroissial du diocèse de Reims à la fin du XVIII ${ }^{\mathrm{e}}$ siècle», Études ardennaises (avril-juin 1967): 22.

56 Histoire de la seigneurie de Lauzon, 4: 238.

57 «Dans sa monographie de la seigneurie de Lauzon, Joseph-Edmond Roy décrit le curé du début du XIX ${ }^{\mathrm{e}}$ siècle comme un prêtre à la vie exemplaire et fidèle au devoir de sa charge. Le curé se recrute, selon lui, chez les «habitants» avec lesquels il reste en contact constant». Pierre Savard, op . cit., 28. Il s'agit d'une réédition de son article «La vie du clergé québécois au XIX siècle», Recherches sociographiques VIII,3 (septembre-décembre 1967): 259-73.
} 
sionnel est couramment fourni dans les tomes subséquents consacrés au clergé encore vivant au moment où Allaire publie son oeuvre, soit le début du XXe siècle. Hamelin s'est probablement laissé convaincre par la lecture répétée de l'étiquette «cultivateur». La consultation systématique de Allaire aurait donc conduit aux conclusions impressionnistes dont il faut remettre en cause la validité pour une période assez longue de l'histoire du Québec.

L'analyse a fait ressortir un certain nombre de similitudes entre les trajectoires socio-religieuses française et québécoise. L'importance accordée aux liens de parenté socio-culturelle aurait-elle indûment relégué dans l'ombre les disparités? Il faudra peut-être un jour faire un bilan plus nuancé des ressemblances et des différences. Ainsi, la chute du recrutement urbain obéit-elle aux mêmes facteurs de part et d'autre de l'Atlantique? La configuration linguistique et religieuse des villes québécoises au cours des années 1820-1840 ne ressemble pas aux situations urbaines françaises. En 1831, plus de la moitié des chefs de familles montréalais sont anglophones; vers 1840 , la proportion voisine $60 \%$; la ville de Québec compte 45\% d'anglophones en 1831. Comme le groupe linguistique anglais est très majoritairement protestant, ce facteur, plus que tout autre peut-être, explique l'absence de vocations urbaines. Mis à part quelques professions comme le notariat, il faut également noter que les anglophones sont surreprésentés dans les strates supérieures de la hiérarchie sociale. Comme l'aisance matérielle ou le capital culturel sont des stimuli à la scolarisation des fils, ces facteurs jouent également contre la fréquentation des collèges par les francophones au cours des décennies où ces derniers voient leur poids numérique grugé par l'immigration anglaise. Au début du siècle, d'autres variables comme la croissance rapide, et partant la jeunesse de la population urbaine ont eu pour effet de réduire les classes d'âge susceptibles d'entreprendre des études théologiques. Ce sont là des éléments de situations peu en rapport avec la structure démographique des villes françaises. Enfin, les études européennes soulignent la haute fécondité sacerdotale de la classe artisanale, thèse que nous avons reprise, chiffres à l'appui, pour l'exemple québécois. Avons-nous la certitude que cet énoncé est conforme à la réalité? D'après une statistique ${ }^{58}$, la ville de Québec au tournant du XIX ${ }^{\mathrm{e}}$ siècle compte 1 à $2 \%$ de professionnels, 15 à $20 \%$ de gens d'affaires, plus de $40 \%$ d'artisans. Si cette répartition s'avère un jour pas trop éloignée de la réalité, il faudra reprendre l'analyse de la fécondité relative des groupes sociaux.

L'analyse qui précède n'est pas le dernier mot de l'historien. L'échantillon des professions cache peut-être une sous-représentation des familles de milieux populaires. A ces séries lacunaires, s'ajoutent

58 Fernand Ouellet, Le Bas-Canada 1791-1840 (Ottawa, Éditions de l'Université d'Ottawa,
1976), 62. Tous les ordres de grandeur évoqués dans ce paragraphe proviennent de cet ouvrage. 
les statistiques démographiques de l'époque qui sont par ailleurs plus ou moins fiables. Elles constituent tout au plus des ordres de grandeur dont il faudra préciser un jour la valeur par de multiples tests de concordance. Pourtant, le danger de constructions «mythiques» ne vient peut-être pas principalement d'erreurs de calcul, si grandes soient-elles. Un postulat philosophique à la mode confère un très haut degré de crédibilité à l'analyse quantitative. Le sociologue Sorokin a désigné du néologisme «quantophrénie» l'abus de l'argumentation statistique et les fausses certitudes qu'il engendre. Voilà pourquoi l'analyse qui postule implicitement une corrélation - et non une coöncidence - entre milieu d'origine et option sacerdotale, élimine peut-être une foule de facteurs notamment «motivationels» à l'origine d'un choix de carrière; celui-ci relève peut-être davantage du libre arbitre que des coordonnées sociogéographiques familiales. Par ailleurs, il se trouve au sein de la population étudiée, quelques sous-groupes dont l'incidence familiale sur le choix de carrière a probablement été nulle. Il en est ainsi de cette dizaine d'hommes à la vocation tardive venus au sacerdoce après avoir pratiqué le droit, ou simplement recouvré la liberté à la mort de leur conjointe. Il existe aussi plusieurs prêtres, orphelins de père depuis leur plus jeune âge. Que signifie pour eux la profession du père comme facteur de dissuasion ou d'incitation à la prêtrise? Ce sont là des questions qui demeurent sans réponse et légitiment l'enquête qualitative à laquelle il faudra soumettre les recrues de l'époque. Néanmoins, la connaissance du milieu d'origine s'avère un précieux point de départ, quelque approximative qu'elle paraisse. 